\title{
KRAS and BRAF mutations induce anoikis resistance and characteristic 3D phenotypes in Caco-2 cells
}

\author{
MADHURA PATANKAR $^{1,2}$, SINIKKA ESKELINEN ${ }^{1}$, ANNE TUOMISTO $^{1,2}$, \\ MARKUS J. MÄKINEN ${ }^{1-3}$ and TUOMO J. KARTTUNEN ${ }^{1-3}$ \\ ${ }^{1}$ Department of Pathology, Cancer and Translational Medicine Research Unit, University of Oulu, 90014 Oulu; \\ ${ }^{2}$ Medical Research Center Oulu, University of Oulu, 90014 Oulu, \\ ${ }^{3}$ Department of Pathology, Oulu University Hospital, 90029 Oulu, Finland
}

Received March 26, 2019; Accepted August 5, 2019

DOI: $10.3892 / \mathrm{mmr} .2019 .10693$

\begin{abstract}
In a number of types of cancer, anoikis, a form of apoptosis induced by loss of extracellular matrix (ECM) attachment, is disturbed. Anoikis resistance is essential in the formation of metastases. A recent study identified carcinoma cell subpopulations surviving without ECM contact in pathological specimens of colorectal cancer. The occurrence of these subpopulations indicated anoikis resistance. In the present study, it is demonstrated that KRAS and BRAF mutations induce anoikis resistance in colon cancer (Caco-2) cells. In 3D cultures, Caco- 2 cells transfected with mutated KRAS or BRAF formed multicellular structures analogous to anoikis-resistant subpopulations in actual carcinomas, and serve as an in vitro model for anoikis resistance. Caco- 2 cell lines were constructed, with KRAS or BRAF mutations, using retroviral delivery. The current study investigated anoikis resistance using an Annexin $\mathrm{V}$ apoptosis test from suspension cultures. 3D in vitro cultures, which were generated in collagen-matrigel mixtures, were assessed using confocal microscopy. 3D cultures embedded in paraffin were analyzed using conventional histopathology. In suspension cultures, Caco-2 cells with KRAS or BRAF mutations indicated a significantly lower proportion of Annexin positivity than the native Caco-2 cells, indicating that these mutations induce anoikis resistance in Caco- 2 cells. 3D cultures displayed native Caco- 2 cells forming polarized cysts with a single layer thick epithelium, whereas Caco-2 cells with KRAS or
\end{abstract}

Correspondence to: Professor Tuomo J. Karttunen, Medical Research Center Oulu, University of Oulu, POB 5000, Aapistie 5, 90014 Oulu, Finland

E-mail: tuomo.karttunen@oulu.fi

Abbreviations: CRC, Colorectal carcinoma; MAPK, Mitogen-activated protein kinases; ERK, Extracellular signal-regulated kinases; MIP, Micropapillary structures

Key words: colorectal cancer, anoikis resistance, Caco-2 cells, KRAS mutation, BRAF mutation, apoptosis
BRAF mutations formed partially filled cystic structures or solid round structures where only the outermost layer was in contact with the ECM. Additionally, KRAS mutations induced reversed polarity to Caco-2 cells along with the emergence of solid growth. The present study demonstrated that KRAS and BRAF mutations induce anoikis resistance in Caco-2 colorectal cancer cells. The growth patterns generated from the KRAS and BRAF mutated cells in 3D cultures revealed a resemblance to the putative anoikis-resistant subpopulations in actual carcinomas, including micropapillary structures and solid tumor cell islands. Additionally, KRAS mutation induced the emergence of inverted polarity. In conclusion, 3D cultures with modified Caco- 2 cells serve as a valid in vitro model for anoikis resistance and inverted polarity.

\section{Introduction}

Colorectal cancer (CRC) is the third most common cancer worldwide (1). In CRCs, 30-50\% carry KRAS mutations, most commonly in codon 12/13 (2), and 10-15\% carry BRAF V600E mutations (3). KRAS mutation, an independent predictor of metastatic CRC, is associated with poor prognosis (4-6). By activating the MAPK/ERK pathway, KRAS mutation influences cell metabolism, increases proliferation, and alters cell death. Similarly, BRAF V600E mutation results in activation of the MAPK/ERK pathway and is highly associated with poorly differentiated CRCs (5-8) and poor prognosis in microsatellite stable CRC (9). However, the most essential biological mechanisms concerning KRAS and BRAF mutation with adverse prognosis are unknown.

Tumor cells show diverse aberrations in the regulation of cell maturation and death mechanisms, which enable uncontrolled growth and metastasis. Anoikis, a programmed cell death mechanism activated in the absence of attachment of cells to an appropriate matrix, is often disturbed in cancer. Inhibition of anoikis during metastasis is an essential mechanism for the formation of metastases (10-12). Anoikis resistance, i.e. anchorage-independent survival of tumor cells, may result from alterations in cell death mechanism, faulty or absent integrin signaling, or through epithelial mesenchymal transition $(13,14)$. There is only limited information available on the role of KRAS and BRAF mutations in anoikis 
resistance. Among colorectal carcinoma cell lines, only HCT116 cells with inherent KRAS mutation are known to show anoikis resistance $(15,16)$. The downstream mechanisms involve both inhibition of apoptosis by SRC activation (15) or downregulation of PHLPP1 and by metabolic regulation (16). Interestingly, codon 12 mutations induce higher level of anchorage-independent survival compared to codon 13 mutations (17), and associate most evidently with adverse prognosis (18). BRAF mutation associates with anoikis resistance in melanoma cell lines and in HT29 intestinal cancer cells (15). BRAF induces anoikis resistance by modulating Bad and Bim signaling in melanoma cells $(19,20)$, whereas in CRC cell lines, MCL1 upregulation via MEK/ERK signaling was identified as a factor for anoikis resistance (21).

Despite the importance of anoikis resistance for metastasis, there have not been many efforts to recognize and quantitate anoikis-resistant subpopulations in actual human cancers. We have recently identified tumor cell subpopulations, such as micropapillary structures (MIPs) in human colorectal carcinomas, which lack contact with the extracellular matrix (ECM) but still show a lower apoptosis rate, indicating that these structures represent an anoikis-resistant subpopulation (22). Interestingly, the low apoptosis rate in these structures was associated with decreased patient survival, supporting the concept that anoikis resistance detected in pathological specimen is prognostically important. Further supporting the importance of anoikis-resistant subpopulations in CRC, we recently observed that abundance of anoikis-resistant subpopulations of carcinoma cells as quantitated by histopathology is an independent marker of adverse prognosis (unpublished).

Although we can study anoikis resistance in vitro with several experimental settings (23-25), these models lack structural relevance in terms of organization of multicellular structures and interaction of cells as in actual tumors (26-30). CRC cell line Caco-2 is a well-characterized tumor cell line forming columnar epithelium-like sheets in two-dimensional cultures, and has the ability to form ball-like structures (cysts) with a fluid-filled lumen surrounded by apical surfaces of epithelial cells in three-dimensional (3D) cultures. KRAS G12V and BRAF V600E mutations induce additional features in native Caco-2 cells such as increased proliferation, alterations in apical-basal polarity, and enhanced migration and invasion properties $(31,32)$. Interestingly, such Caco-2 cells with KRAS or BRAF mutations form solid cell clusters in 3D cultures $(31,32)$. Since the inner cells within such clusters might be devoid of extracellular matrix, unlike the outer cells, which rest in the semisolid 3D culture medium with ECM $(31,32)$, such cells in 3D cultures could possibly serve as a model for the anoikis-resistant subpopulation of carcinoma cells seen in actual human cancers. However, it is unknown whether KRAS or BRAF mutations induce anoikis resistance in Caco- 2 cells.

To study the involvement of KRAS and BRAF mutations in anoikis resistance in colorectal carcinoma cells in vitro and to develop an in vitro model for multicellular clusters occurring in actual carcinomas in vivo that corresponds to anoikis resistance, we generated modified Caco-2 cell lines with KRAS G12V or BRAF V600E mutations. We studied the growth patterns of these cell lines in $3 \mathrm{D}$ cultures in vitro and in suspension cultures for anoikis resistance and found evidence for the emergence of anoikis resistance and for structural analogies with anoikis-resistant populations in human CRC. Finally, we evaluated the role of Bim in the anoikis resistance of Caco-2 cells.

\section{Materials and methods}

Transfection of Caco-2 cells with mutated KRAS and BRAF Production of retroviral supernatants. Phoenix cells were cultured in 6-well cell culture plates in DMEM media with $10 \% \mathrm{FBS}$ and antibiotics at $37^{\circ} \mathrm{C}$ in $5 \% \mathrm{CO}_{2}$. At confluency, cells were transfected with Vsvg and retroviral vectors (pQCXIP GFP, pQCXIP GFP-K-Ras V12 (G12V), pQCLAP GFP-Braf-V600E) for retroviral production; the plasmids were a kind gift from Professor Alan Hall, Memorial Sloan-Kettering Cancer Center, New York, USA. In a $1.5 \mathrm{ml}$ Eppendorf tube, $(1 \mathrm{ml})$ OptiMEM media per transfection with additional $50 \mu \mathrm{l}$ Lipofectamine 2000 agent was prepared by gently mixing, followed by 5 -min incubation at room temperature. In another $1.5 \mathrm{ml}$ tube, $24 \mu \mathrm{g}$ of respective retroviral vector and $2.5 \mu \mathrm{g}$ of pVsVg envelope plasmid per transfection were mixed in a $250 \mu$ l optimum tube. We incubated the Lipofectamine mix combined with DNA for $20 \mathrm{~min}$, added this mixture dropwise on cells with gentle swirling, and placed the cells back into $37^{\circ} \mathrm{C}$ incubator overnight. After harvesting viruses, we collected the supernatant for two consecutive days in $15 \mathrm{ml}$ tubes to a total volume of $10 \mathrm{ml}$. For good viral titer, we concentrated the supernatant by ultracentrifugation $\left(16,500 \mathrm{rpm}, 2 \mathrm{~h},+4^{\circ} \mathrm{C}\right)$. The pellet was carefully suspended in PBS and either used for infections or stored at $-80^{\circ} \mathrm{C}$.

Infection and selection of cells. $1.5 \times 10^{5}$ Caco-2 cells (from ATCC) were plated in 6-well cell culture plates (Corning) and infected with concentrated virus supernatants supplemented with polybrene and additional 500 yp of complete MEM medium. After infecting the cells twice, we selected clones with respective antibiotic selection (Puromycin $4 \mu \mathrm{g} / \mathrm{ml}$, Sigma-Aldrich; G418-0.8 mg/ml, Merck) from the third day post-infection. We sorted $20 \%$ stronger (Fluorescence mean intensity) GFP positive clones with BD FACS Aria Illu (BD Biosciences) with appropriate machine settings, plated them in 6 well plates and expanded. In addition, we assessed the presence of BRAF and KRAS constructs in Caco2-BRAF and Caco2-KRAS cell lines by western blot analysis with rabbit polyclonal anti-GFP antibody at dilution 1:1,000 (A11122, Invitrogen, UK) and BRAFV600E mouse monoclonal at dilution 1:1,000 (VE1, Roche Diagnostics, Indianapolis, IN, USA) (Fig. S1).

$3 D$ cell culture. Caco2-GFP cells (Caco2-control), Caco2-GFP cells expressing KRAS G12V (Caco2-KRAS), and Caco-GFP cells expressing BRAF V600E (Caco2-BRAF) were cultured in DMEM media supplemented with sodium pyruvate (Sigma), $15 \%$ FBS, $1 \%$ antibiotics and 1\% NEAA (Sigma), referred to as DMEM complete medium, and cultured at $37^{\circ} \mathrm{C}$ in $5 \% \mathrm{CO}_{2}$. Cells were cultured in 3D cultures mainly as described by Debnath et al (33) with addition of a mixture of Matrigel and Collagen at a ratio of 4:1 instead of matrigel alone as described by Magudia et al (32).

For live cell imaging of 3D structures, $35 \mathrm{~mm}$ glass bottom dishes (Greiner Bio-One) were coated with $80 \%$ 
matrigel (growth factor reduced, Sigma) and 20\% Collagen 1 Rat tail (Life Technologies) on ice. We plated 70,000 cells on pre-coated dishes with $500 \mu$ l DMEM complete medium supplemented with $2 \%$ matrigel (referred to as food). For fixed 10-day-old 3D structures, we coated 4 well-chambered slides (Thermo Fisher Scientific) with $80 \%$ matrigel and 20\% collagen I mix on ice. We plated single cell suspension of $10^{4}$ cells on precoated slides with an additional $200 \mu 1$ of food. Food was added every second day to all of the 3-D cultures.

Staining and imaging of $3 D$ cell cultures for microscopy. For live imaging, 3D structures grown in $35 \mathrm{~mm}$ glass bottom dishes were washed once with media, incubated with complete DMEM media with $1 \mathrm{mg} / \mathrm{ml}$ Hoechst 33342 dye (Thermo Scientific) at $37^{\circ} \mathrm{C}$ for $30 \mathrm{~min}$, and transferred to illumination chamber of Zeiss Spinning Disk Confocal microscope set at $37^{\circ} \mathrm{C}$. Images were collected with $0.6 \mathrm{um}$ step size using $40 \mathrm{X}$ objective and excitation wavelengths of $405 \mathrm{~nm}$, EC Plan NeoFluor 40X/0.75 DIC air objective and BP 450/50 nm (blue) emission filters. After imaging, we washed the dye away from the cells, added fresh food to the dishes, and replaced them in the incubators at $37^{\circ} \mathrm{C}$. One dish was imaged for 2 consecutive days and then discarded to limit the effects of stress on the cells during analysis. We collected images for 5-15 cysts from each sample using a spinning-disk confocal microscope and performed each experiment three times. We analyzed and exported images with Huygens Pro, Zen software.

Imaging of fixed $3 D$ structures. The 3D structures were grown for 10 days and fixed with 2\% paraformaldehyde (PFA) in PBS for 15 min blocked with $0.1 \%$ BSA, $0.2 \%$ Triton X $100,0.05 \%$ Tween and 10\% FBS for $20 \mathrm{~min}$ and incubated with TRITC-phalloidin (1:10, Invitrogen) and dapi (1:1,000, Thermo Scientific) for $1 \mathrm{~h}$. We mounted the specimens with Immu-Mount (Thermo Fisher Scientific) after washes with PBS and distilled water. We assessed the specimens with Olympus Flow view Confocal Microscope and collected z-stack images with an Olympus FluoView laser scanning confocal microscope using UPLSAPO 60x/1.35 oil immersion objective, and excitation wavelengths of 405 and $543 \mathrm{~nm}$ and appropriate emission filters were used for dapi and TRITC phalloidin, respectively.

The 3D cell growths were classified as cysts if they had an outer wall formed by a single layer of cells and a lumen without cells or cell debris. The structures filled completely or partially with cells without a clear lumen as in the cysts were identified as solid growths or filled structures. Quantitation of the structures was done manually using $20 \mathrm{X}$ objective by classifying and counting 145-212 structures from fixed 3D specimens. The proportion of 3D structures formed by each cell line was calculated. We performed a minimum of three independent experiments and used the average proportion for graphical representation.

Suspension culture for anchorage-independent survival assay. To make an anchorage-independent surface, $0.5 \mathrm{~g}$ poly-HEMA (Sigma) was added to $25 \mathrm{ml} 95 \%$ ethanol, and the solution was prepared by keeping the flask in $58^{\circ} \mathrm{C}$ water bath for $6 \mathrm{~h}$ and mixing by shaking the flasks every $40-60 \mathrm{~min}$. We plated dissolved poly-HEMA solution in $10 \mathrm{~cm}$ bacterial cell culture dishes, spread evenly and dried overnight under the laminar hood, followed by an additional incubation under UV for $30 \mathrm{~min}$. The prepared plates were used directly for experiments or sealed with parafilm and stored at $4^{\circ} \mathrm{C}$ for up to one week.

For flow cytometry, we plated $1.0 \times 10^{7}$ cells from single cell suspension obtained from trypsin treatment on poly-HEMA coated dishes. After adding $9 \mathrm{ml}$ of serum-free DMEM medium plates were incubated at $37^{\circ} \mathrm{C}$ in an incubator. We performed the apoptosis assay at two time points, 24 and $48 \mathrm{~h}$. On the day of Annexin V test, cells were washed twice with cold Biolegend's Cell Staining Buffer (Biolegend, San Diego, CA, USA), and the pellet was suspended in Annexin V binding buffer (Biolegend, San Diego, CA, USA). For Annexin FACS assay, $100 \mathrm{ul}$ cell suspension was pipetted in a test tube with $5 \mu \mathrm{l}$ BD HorizonTM V500 Annexin V (BD Biosciences, Franklin Lake, NJ, USA), mixed by vortexing, and incubated for $20 \mathrm{~min}$ in dark at room temperature. An additional $400 \mu \mathrm{l}$ Annexin binding buffer (Biolegend, San Diego, CA, USA) was added to the tubes, measured by BD LSR Fortessa (BD Biosciences, Franklin Lakes, NJ, USA) flow cytometer, and analyzed by FACS Diva version 7.2 (BD Biosciences, Franklin Lakes NJ, USA) and FlowJo X software. In addition, we estimated the cells at s-phase indicating proliferative phase (34) using Propidium iodide FACS assay from Caco-2 control cells and Caco2 cells with KRAS or BRAF mutations grown in suspension for $24 \mathrm{~h}$.

Protein expression analysis. We sorted suspension cultures at 24 and $48 \mathrm{~h}$ for Annexin-V negative cells by Flow cytometry (BD FACS Aria) and lysed the cells by sonication in $1 \mathrm{ml}$ radio immunoprecipitation assay (RIPA) buffer consisting of $50 \mathrm{mM}$ Tris-HCL (pH 7.4), $150 \mathrm{mM}$ Nacl, 4 mM EDTA, 1\% NP-40

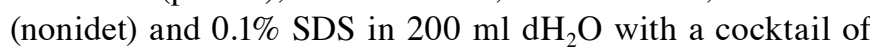
proteinase inhibitors. Lysates were incubated on ice for $30 \mathrm{~min}$ with vortexing every $5 \mathrm{~min}$ and cleared by centrifugation $\left(10,000 \mathrm{~g}, 2 \mathrm{~min},+4^{\circ} \mathrm{C}\right)$. We measured protein concentration by Bradford protein assay (Bio-Rad). For western blot analysis, equal amounts (20 $\mu \mathrm{g})$ of protein samples were separated on $15 \%$ SDS-PAGE and electro-blotted to Nitrocellulose membrane for $2 \mathrm{~h}$ at $+4^{\circ} \mathrm{C}$. We blocked the membrane in $5 \%$ skim milk in TBS-Tween buffer $(1 \mathrm{~h})$ followed by washes with 1x TBS-T buffer and incubation with primary antibody 1:1,000 (Bim Rabbit mAb, C34C5, cell Signaling Technology; Mouse monoclonal Tubulin, 1:5,000) overnight at $+4^{\circ} \mathrm{C}$. After washes, we incubated membranes in HRP conjugated secondary antibody (anti-rabbit, 1:5,000; anti-mouse, 1:10,000) for $1.5 \mathrm{~h}$. We then detected antigen by incubating the membrane for $2 \mathrm{~min}$ in detection solution comprising $250 \mathrm{mM}$ Luminol, $90 \mathrm{mM}$ Cumoric, $3 \mu 1 \mathrm{H}_{2} \mathrm{O}_{2}$ in $10 \mathrm{ml} 0.1 \mathrm{M}$ Tris-HCL ( $\mathrm{pH}$ 8.3), exposed using a chemiluminescence Fuji-Las-3000 detection system (FujiFilm, Minato, Tokyo, Japan). The bands were quantified with Quantity One Analysis software and further calculations and graphs were generated with Microsoft office applications. The Bim expression band intensities were normalized by using those of Tubulin, and fold changes of Bim expression as compared with the expression level in native Caco-2 cells at $24 \mathrm{~h}$ were calculated. 


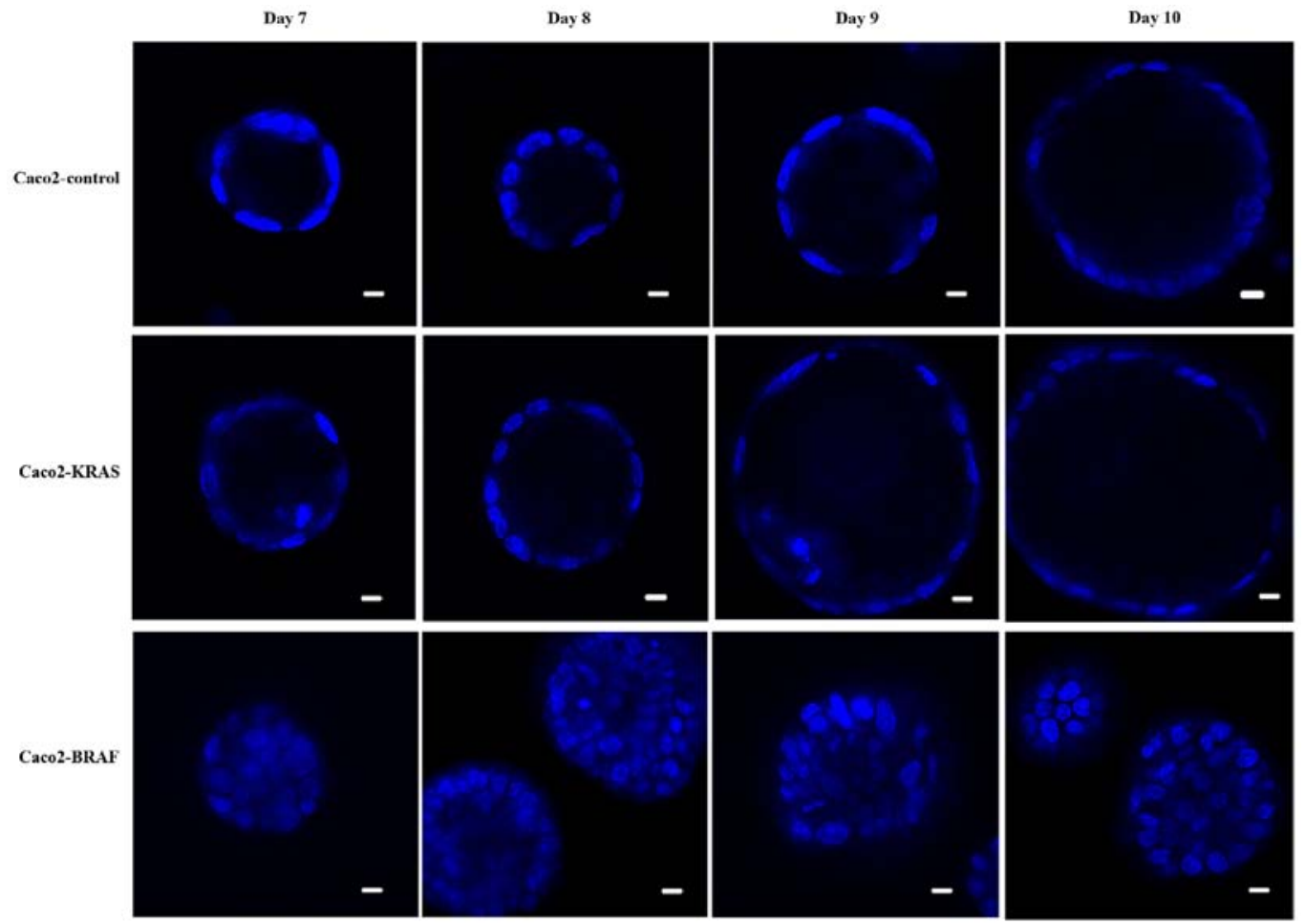

Figure 1. Time-course development from native and KRAS or BRAF mutated colon cancer cells in 3D cultures. The evolution of clusters from day 7 to 10 , using Hoechst (nuclear) staining. Control Caco-2 cells formed cysts, while focal cell piling, occasionally accompanied by apoptosis (Day 9), is present in Caco-2-KRAS cells, and Caco-2-BRAF cells show formation of solid structures. Scale bar represents $20 \mu \mathrm{m}$. 3D, 3-dimensional.

Paraffin embedding of $3 D$ cell cultures. For this experiment, we modified the protocol of Pinto et al (35). 3D structures were obtained from Caco 2-control, Caco 2-KRAS and Caco 2 -BRAF cell lines cultured in $80 \%$ matrigel $+20 \%$ collagen gel mix for 8 days. On the 8 th day, we washed the cultures once with media. The gel including the 3D cell structures within was detached with a clean razor blade and placed in a cryomold pre-coated with $120 \mu \mathrm{l}$ hot agarose gel. We added an additional $120 \mu \mathrm{l}$ hot agarose gel on top of the specimen, forming a sandwich. The mold was placed on ice to solidify, transferred into pathology cassettes, and fixed in $10 \%$ formaldehyde overnight, and then washed with running water, dehydrated with increasing alcohol concentration (70\% ethanol, $96 \%$ ethanol, $100 \%$ ethanol), xylene, and finally, embedded in paraffin. The paraffin blocks were stored at room temperature until further use.

For imaging and analysis of paraffin-embedded 3D cultures, $4 \mu \mathrm{m}$ sections were cut from the paraffin blocks and stained with hematoxylin and eosin. We analyzed the slides manually under a light microscope and recorded the observation.

Statistical analysis. We assessed significances of the differences between the cell lines with ANOVA and used Tukeys correction in post hoc analyses. We used IBM SPSS 25 (IBM, Armonk, NY, USA).

\section{Results}

Transfection with mutated KRAS or BRAF influences morphology of $3 D$ structures of Caco-2 cells. We cultured conventional Caco-2 cell line (controls) and Caco-2 cells with either KRAS or BRAF mutation in a mixture of matrigel and collagen for up to 10 days. Live cell imaging indicated that during the initial days, the cells proliferated and formed balls of cell clusters growing and expanding within the matrix. Later on, i.e. post the 8th day, most Caco-2 control cells formed round cysts with an epithelial cell lining around a fluid-filled lumen (Fig. 1). The structures formed by Caco2-KRAS and Caco2-BRAF cell lines were larger than those formed by the control cells. In addition, some structures formed by Caco2-KRAS cells were cysts similar to those seen in Caco-2 controls (Fig. 1) with a clear lumen while others formed cystic structures partially filled with cells. Finally, some cysts formed by Caco2-KRAS cells showed focal piling up of cells (Fig. 1) or apoptotic cells in the luminal space adjacent to cyst wall (Fig. 1). In contrast, Caco2-BRAF cells formed predominantly solid structures with cells filling up the lumen (Fig. 1), an appearance resembling solid tumor cell clusters in some of the clinical carcinoma specimens. The growth patterns were consistent in terms of cyst formation and solid growth from the 7th day onwards in both control and KRAS or BRAF mutated 3D cultures until the 10th day (Fig. 1).

To understand the organization and polarization of cells in 3D cultures, we imaged 10-day-old cultures after fixation and staining with TRITC-phalloidin and DAPI. Caco-2 control cells showed regular apical-basal polarity as indicated by regular apically polarized localization of TRITC-phalloidin staining (Fig. 2A). In contrast, Caco2-BRAF cells showed irregular polarity with irregular strands of phalloidin present between cells within the clusters (Fig. 2B). Caco2-KRAS cells presented variable irregularity of cell polarity in the cell clusters. Here, the clusters with only focal cell piling up 

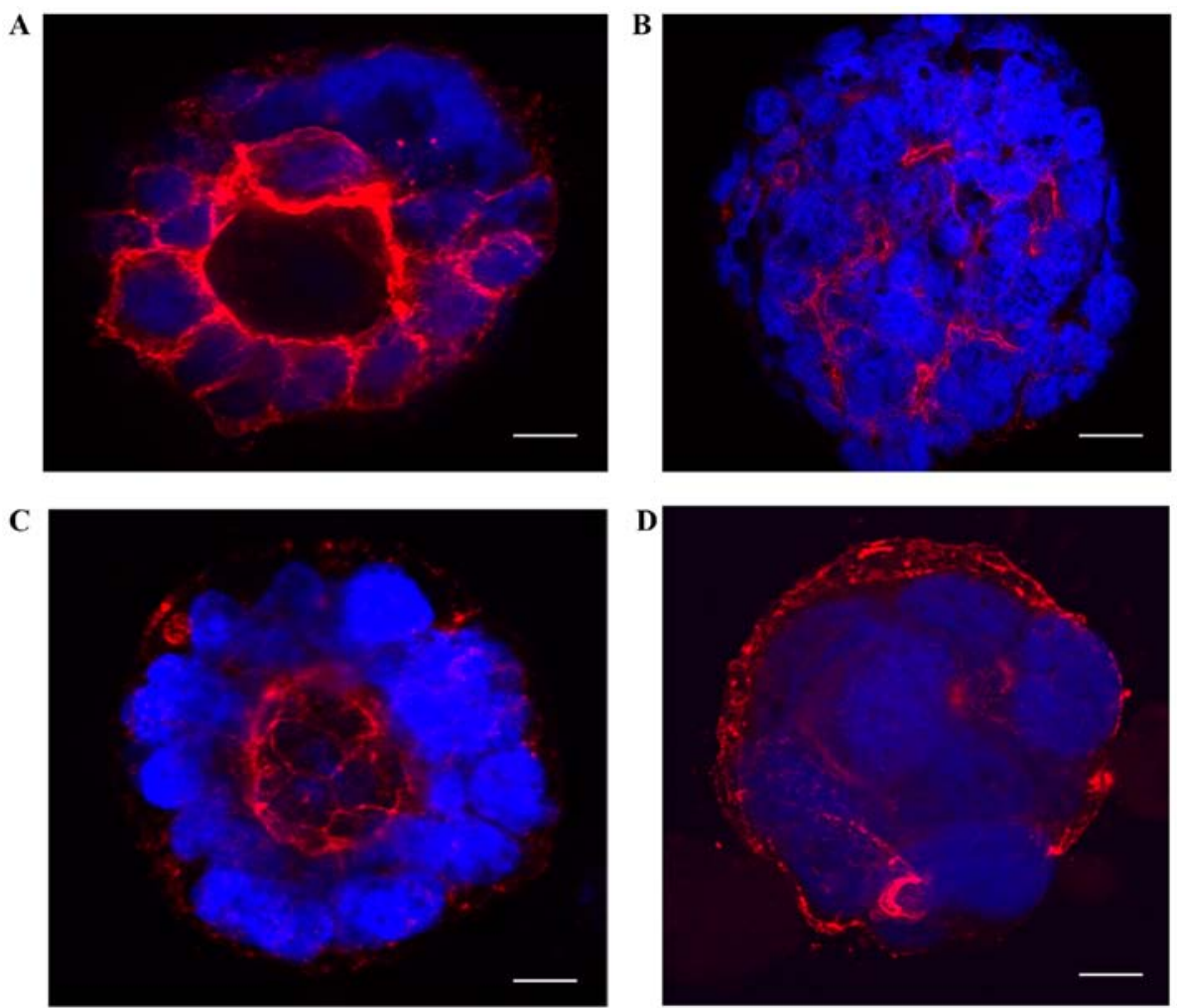

Figure 2. Structural differences between native Caco-2 cells and Caco-2 cells transfected with mutated KRAS or BRAF. Confocal images of 10 day old 3D cell cultures, fixed and stained with DAPI (nuclear), TRITC-phalloidin (actin filaments). (A) Native Caco-2 cells formed cysts with retained apical-basal polarity of the cells as presented by regular polarized location of strong continuous actin staining at apical side. (B) Caco-2-BRAF cells showed solid structures with distorted actin staining and highly irregular polarity. Caco-2-KRAS cells showed two growth patterns. (C) Weak, irregular, focally absent apical staining for actin along with irregular polarity and some piling up of the cells was seen. (D) Cells formed 3D structures with filled lumen and irregular polarity, with notable actin staining in the most superficial parts of the cell cluster, and this organization indicating inverted polarity. Scale bar represents $20 \mu \mathrm{m}$. 3D, 3-dimensional.

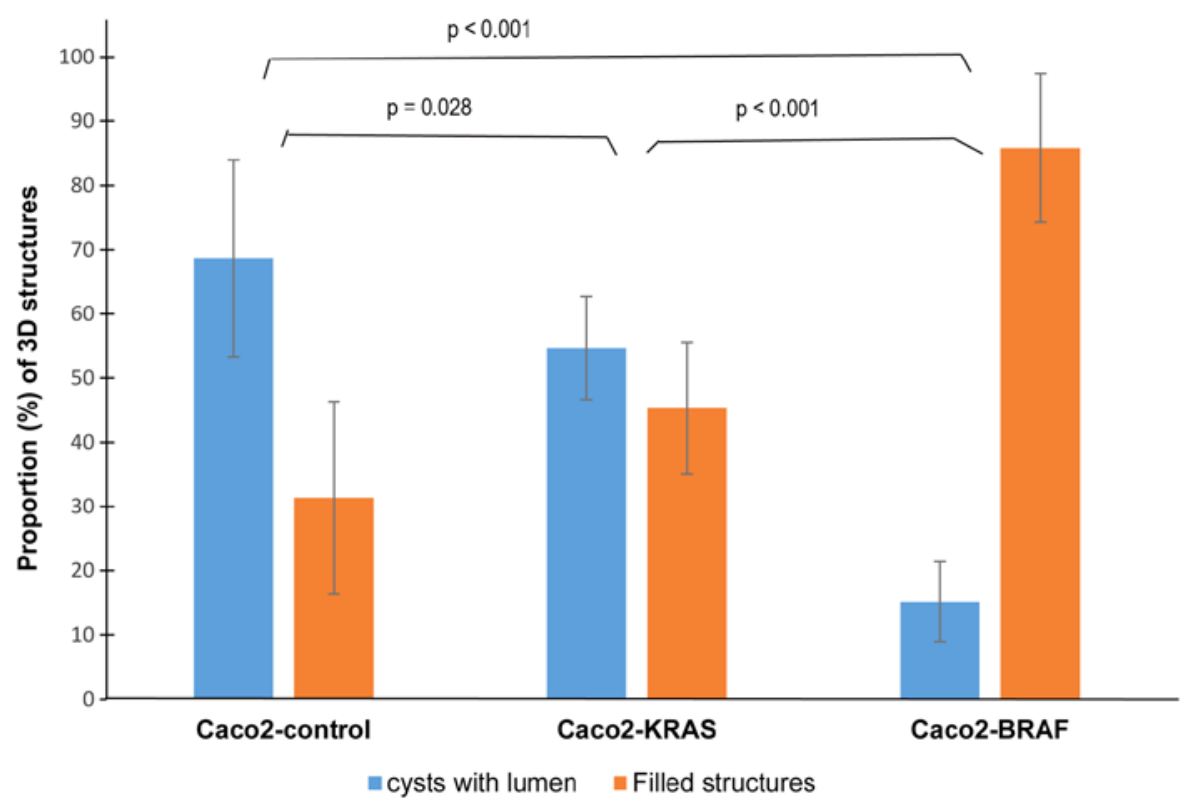

Figure 3. Quantification of 3D structures. This is a graphical representation of 3D structures formed by control Caco-2 and Caco-2 cells with KRASor BRAF mutations at day 10. Columns represent average proportions $(\%)+$ - standard deviation of 3D structures formed from each cell line, from three independent experiments. 3D, 3-dimensional.

displayed regular apical-basal polarity (Fig. 2C). In contrast, Caco2-KRAS cells also formed solid clusters with an inverted polarity pattern, shown by actin staining close to the outer surface of the clusters (Fig. 2D).
Since the cystic or solid growth patterns did not completely relate with the cell lineage, we quantitated different growth patterns in the 10-day-old fixed culture specimens. The majority (about $70 \%$ ) of the structures formed by the control Caco-2 


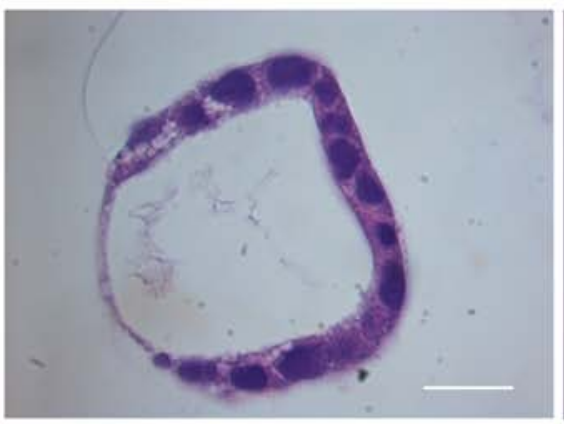

Caco-2 control

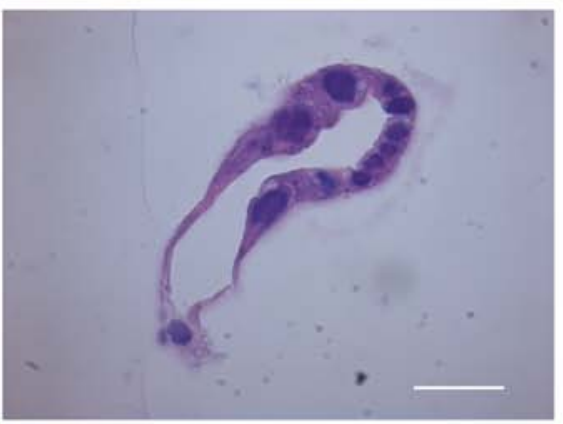

CaCO2-KRAS

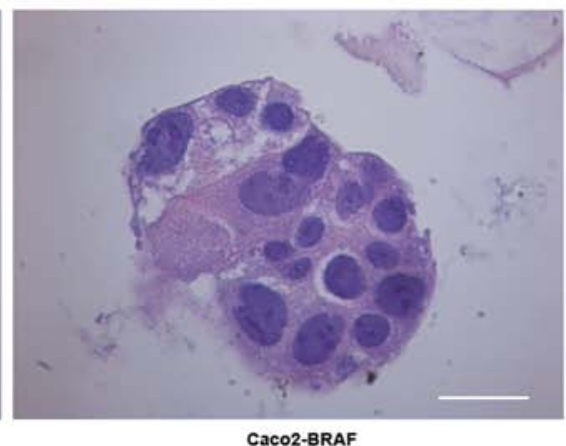

Caco2-BRAF

Figure 4. Microphotographs from Hematoxylin \& Eosin stained paraffin-embedded 3D cultures. The figure panel indicates morphological differences between Caco-2 cells (Caco2-control), Caco-2 cells with KRAS (Caco2-KRAS) or BRAF (Caco2-BRAF) mutations. Scale bar=20 $\mu$ m. 3D, 3-dimensional.

A
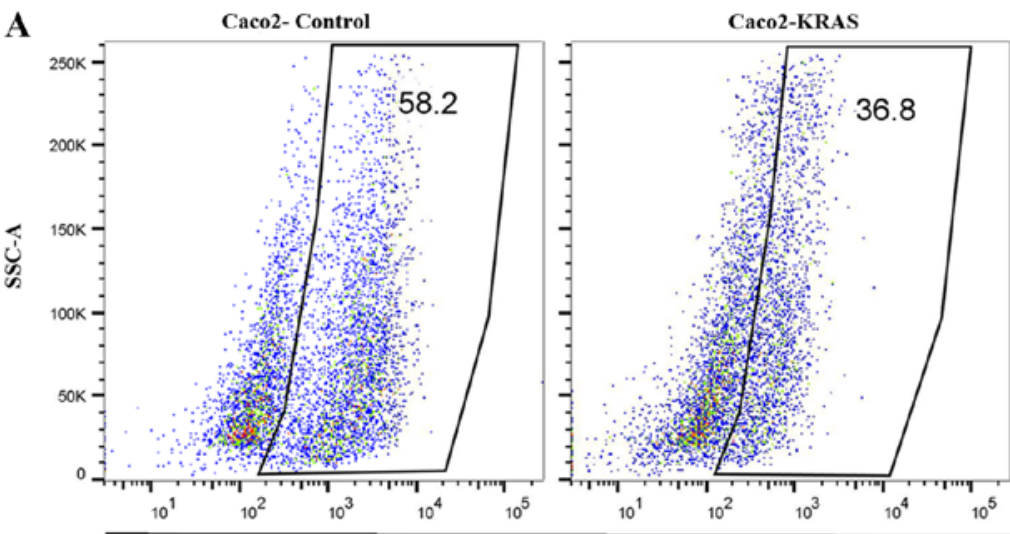

Caco2-KRAS

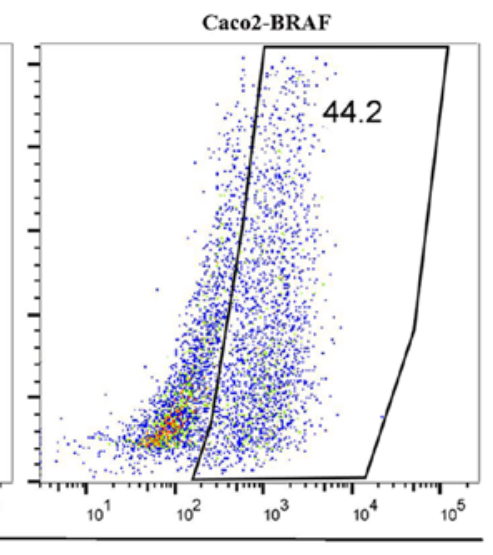

Annexin V_V500

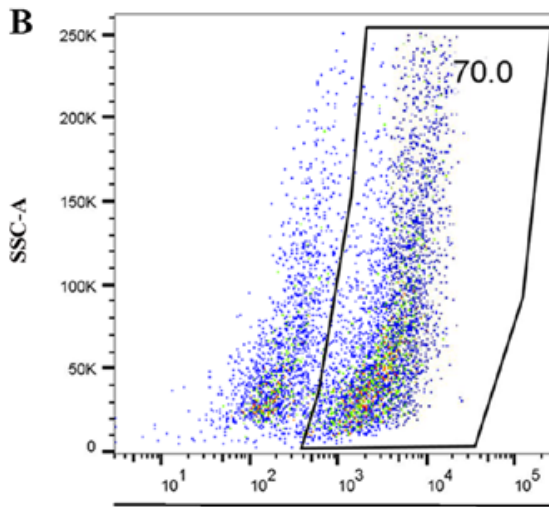

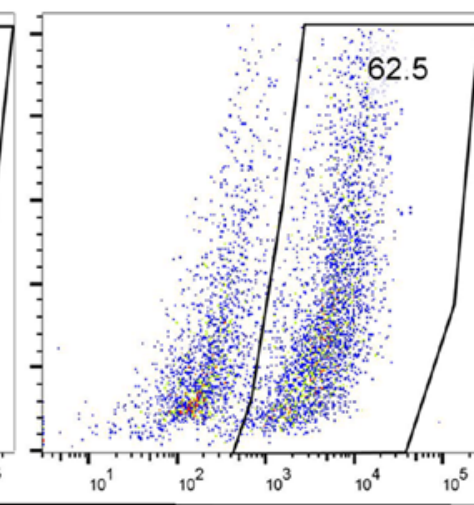

Annexin V_V500

Figure 5. Apoptosis assay from suspension cultures. The scatter plots showing proportions of annexin positive cells (apoptotic cell \%) from Caco2-controls, Caco2-KRAS and Caco2-BRAF suspension cultures at (A) $24 \mathrm{~h}$ (upper row) and at (B) $48 \mathrm{~h}$ (lower row). Boxes represent annexin positive cells and the numbers indicate their proportions. SSC-A, side-scatter area plot.

cells were cysts, with occasional solid structures (Fig. 3). In Caco2-KRAS 3D cultures, about half of the structures were solid and the other half were similar to control Caco-2 cysts, whereas Caco2-BRAF cultures dominantly consisted of solid structures.

Conventional light microscopy study of $3 D$ cell cultures embedded in paraffin. We cultured a new batch of Caco-2 control cells, Caco2-KRAS and Caco2-BRAF cells in matrigel-collagen mix for 8 days. Quick embedding of 3D specimens into paraffin allowed long-term storage of the specimens and comparison with conventional histopathology of actual tumor specimens. Sections stained with hematoxylin and eosin presented similar structural differences between the cell lines as observed by live imaging experiments (Figs. 1 and 4), with structures resembling MIPs (22) and solid cell clusters seen in clinical colorectal carcinoma specimens.

KRAS or BRAF mutations and anchorage-independent survival of Caco-2 cells. To assess the influence of mutations on the anchorage-independent survival ability of colon cancer cells, we cultured Caco2-control cells, Caco2-KRAS and Caco2-BRAF cells on poor attachment (poly-HEMA coated) 


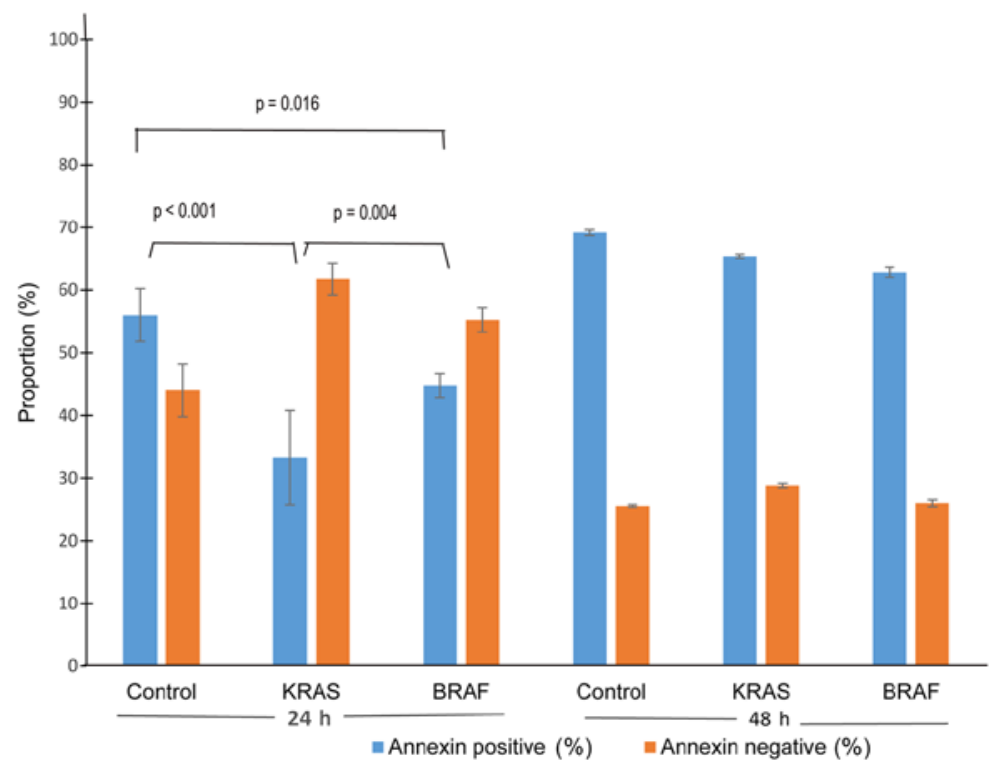

Figure 6. Graphical overview of anchorage-independent survival in Caco2-control cells, Caco2-KRAS and Caco2-BRAF cells. Proportions (\%) of Annexin positive (apoptotic) and Annexin negative (surviving) from native Caco-2 cells (controls), Caco-2 cells with KRAS mutation and Caco-2 cells with BRAF mutation. The graph comprises mean values $+/$ - standard deviation of three independent experiments.
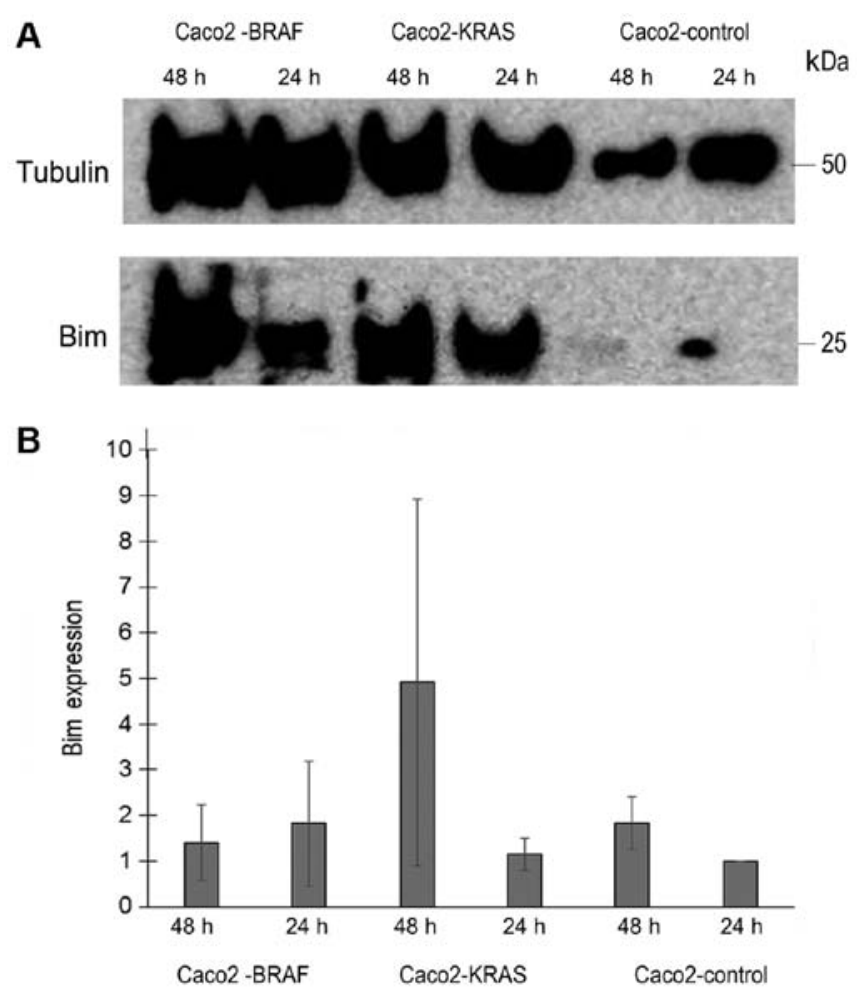

Figure 7. Bim expression in native Caco-2 cells, and in Caco-2 cells transfected with KRAS V12 or BRAF V600. After $24 \mathrm{~h}$ or $48 \mathrm{~h}$ culture in suspension, Annexin negative cells were sorted lysed and analyzed for Bim expression level by western blotting. Tubulin was used for normalization. (A) Shows a representative western blot. (B) Shows mean values of normalized expression of Bim based on three experiments, relative to (fold change) expression in native Caco-2 cells at $24 \mathrm{~h}$. Lines indicate standard error of the mean. There were no statistically significant differences between the cell lines.

surfaces and studied anoikis resistance. Annexin V500 assay showed significantly lower apoptosis rate in Caco2-KRAS and Caco-2-BRAF cells than in Caco-2 control cells at $24 \mathrm{~h}$, whereas cells grown in suspension for $48 \mathrm{~h}$ presented increased apoptosis rate independent of cell type (Figs. 5 and 6). In addition, we determined S-phase fractions to get impression of proliferation activity of the cells in anchorage independent conditions. At $24 \mathrm{H}$, the S-phase fraction in control Caco-2 cells tended to be higher $(n=1 ; 25.1 \%)$, as compared to cells transfected with KRAS ( $n=2$; mean $14.7 \%$, SD 0.6), and in cells with BRAF ( $n=3$; $22.0 \%$, SD 1.3), but at $48 \mathrm{~h}$ all cell lineages showed practically similar S-phase fractions (25.4; 24.6; 23.2\%, respectively).

To assess the mechanism behind the anoikis resistance by KRAS or BRAF mutations in Caco- 2 cells, we analyzed Bim expression level in lysates from annexin negative Caco-2 control, Caco2-KRAS and Caco2-BRAF cells, cultured in suspension for $24 \mathrm{~h}$ or $48 \mathrm{~h}$. Based on three similar experiments, we saw evidence for Bim upregulation in Caco-2 cells transfected with mutated KRAS and BRAF, which was not statistically significant. For KRAS transfected cells, the increase was most evident at $48 \mathrm{~h}$ with more than two fold increase than control Caco-2 cells at the same time point. For BRAF transfected cells increase was 1.8 fold at $24 \mathrm{~h}$ (Fig. 7).

\section{Discussion}

Anoikis resistance is an essential feature of malignant cells. Without it, cancer cells detached from their primary site would die during their travel through lymphatic or blood vessels or tissue cavities $(11,14)$. So far, it has only been possible to analyze anoikis resistance with in vitro cell culture experiments. However, according to our recent observations, anoikis resistance correlates to microscopical features of human carcinomas, such as occurrence of cell subpopulations without ECM contact (22). However, there have not yet been any in vitro models for anoikis resistance where combining actual anoikis resistance of the cells and formation of multicellular structures would mimic the features comprised in 
Table I. Review of reported 3D growth patterns and anoikis resistance in native Caco-2 cells, Caco-2 cells transfected with KRAS or BRAF mutations (present study), and intestinal carcinoma cell lines with inherent KRAS or BRAF mutation.

\begin{tabular}{lllll}
\hline Cell line & KRAS or BRAF mutation & 3D growth pattern & Anoikis resistance & \multicolumn{1}{c}{ (Refs.) } \\
\hline Caco-2 & None & Polarized cyst & NS & $(32,52)$ \\
Caco-2 & None & Polarized cyst & + & Patankar M (Present study) \\
Caco2-KRAS & KRAS, G12V & Solid & NS & $(32)$ \\
HCT 116 & KRAS, G13D & Solid & NS & $(15,53-55)$ \\
& & NS & + & \\
SW 408 & KRAS, G12V & Solid & NS & $(52,54)$ \\
Caco2-KRAS & KRAS, G12V & Solid & + & Patankar M (present study) \\
Caco2-BRAF & BRAF, V600E & Solid & NS & $(32)$ \\
HT29 & BRAF, V600E & Solid & NS & $(15,53,54)$ \\
& & NS & + & \\
DLD-1 & BRAF, V600E & Solid & NS & $(53,54)$ \\
Caco2-BRAF & BRAF, V600E & Solid & + & Patankar M (present study) \\
\hline
\end{tabular}

3-D, 3 dimensional; NS, not studied; +, studied.

actual tumors with anoikis-resistant cell subpopulations. We present here novel evidence that Caco-2 cells transfected with oncogenic mutants of KRAS or BRAF gene gain anoikis resistance, which is detectable in both conventional in vitro test for anoikis resistance and in 3D cultures. Importantly, 3D cultures showed structural features consistent with anoikis resistance similar to actual carcinomas. These models serve for analyzing mechanisms by which KRAS and BRAF oncogenic mutations support anoikis resistance and for studying the general mechanisms of the formation and organization of multicellular groups with activated anoikis resistance mechanisms. In addition, the 3D model mimics actual tumors consisting of two subpopulations of cells, one in contact with the ECM and the other without such contact to the matrix along with activated anoikis resistance mechanisms. The model allows analyses of whether these populations differ in responses to factors like hypoxia or to treatment modalities such as radiation and oncological drugs.

For our model, we used Caco-2 cells, a well-characterized intestinal cancer cell line forming regular monolayers and showing sensitivity for anoikis (36). Caco-2 was also an optimal cell line since without mutations, the growth and functions are very close to normal colorectal/intestinal epithelium and there is no inherent anoikis resistance in this cell line (36). To assess acquisition of anoikis resistance, we selected mutated KRAS (G12V) and BRAF (V600E) oncogenes owing to their clinical and biological relevance in colorectal adenocarcinoma. Besides being common in CRC $(2,3,37,38)$, these mutations have prognostic significance and have importance in the selection of treatment modalities (4-6,9). Importantly, these mutations induce solid growth instead of polarized cysts in 3D cultures $(31,32)$, such growth possibly indicating induction of anoikis resistance. We confirmed transfection efficiency by respective antibiotic selection, by sorting GFP positive cells by flow cytometry, and by confirming the expression GFP/KRAS and GFP/BRAF constructs by western blots.
We confirmed emergence of anoikis resistance in Caco-2 cells transfected with mutated KRAS or BRAF genes by two complementary sets of experiments. First, both Caco2-KRAS and Caco2-BRAF cells showed enhanced anoikis resistance as compared with native Caco-2 cells, cells transfected with mutated KRAS showing higher anoikis-resistant survival than those transfected with mutated BRAF (Figs. 5 and 6) in the anti-adhesion tests. In these tests, the differences between control and KRAS or BRAF transfected cells were evident after $24 \mathrm{~h}$, but considerably lower in the 48-h samples, possibly due to stress from culturing on anti-adhering surfaces and the long incubation period. A limitation in our apoptosis assay was the lack commonly used Propidium iodine (PI) staining accompanying Annexin staining. However, the focus of present work was anoikis resistance and Annexin V staining reveals cells from early to late apoptosis. Since PI staining is an indicator of progress of apoptosis and of non-apoptotic cell death, it would not have provided more information on anoikis resistance. Theoretically, reaction by hyper proliferative state to loss of matrix contact is a potential mechanism to overcome anoikis (11). In our experiments, however, transfected cells showed lower (KRAS) or similar (BRAF) proliferation rates as compared with native Caco-2 cells, suggesting that this mechanism does not explain anoikis resistance as detected in anti-adhesion test. In contrast, KRAS might even induce dormancy. As a second complementary evidence for anoikis resistance, in 3D cultures from Caco-2 cells transfected with mutated KRAS or BRAF presented formation of cysts with focal piling up of the cells on the luminal side or formation of solid, completely non-cystic growth, with inner cells without matrix contact. We saw occasional apoptotic cells among the latter cells, but majority of the cells were surviving (Fig. 1). Occurrence of such matrix-independent survival in 3D cultures indicates anoikis resistance.

Both KRAS and BRAF mutations seemed to induce characteristic patterns in 3D structures in Caco-2 cells. Quantitative analysis of our 3D cultures indicated that native Caco-2 cells mostly formed cysts with a single layer of 
epithelial cells regularly polarized with the apical side facing the cyst lumen. In contrast, Caco2-KRAS cells often (about half of the clusters) formed solid round structures without lumen, while Caco2-BRAF cells mainly formed solid round cell clusters (Figs. 1 and 2). A closer look at the inner cells within these 3D structures revealed that they were in close contact with each other and with the outermost cells of the clusters and showed evidence of irregular cell polarity (Fig. 2). Such clusters may result from disturbed cell adhesion regulation (39) and disrupted integrin signaling. The absence of specific integrin's such as $\alpha 2$ and $\beta 1$ has been shown to lead to irregular apical-basal polarity in epithelial MDCK cells and Caco-2 cells $(39,40)$.

While both BRAF and KRAS mutations induced irregular polarity in the inner cells of Caco- 2 clusters, only KRAS induced dislocation of actin staining close to the outer surface of the cell clusters, a pattern indicating development of inverted polarity (32). A recent report shows the importance of inverted apical-basal polarity in the peritoneal dissemination and invasion of CRC, characteristically present in the serrated subtype of CRC (41). This is of interest as KRAS mutation is the most frequent mutation type and present in $45 \%$ of serrated carcinomas (3). These findings support the role of KRAS mutation in the development of inversed polarity. The current $3 \mathrm{D}$ model is potentially useful in studying the developmental mechanisms and biological significance of inverted polarity.

When compared with structures seen in human CRCs, the 3D structures formed by Caco- 2 cells with KRAS or BRAF mutations demonstrate strong similarities to cell groups without contact to the extracellular matrix. These structures included MIPs (22), solid cell islands, and cribriform structures (unpublished). Solid structures formed by Caco2-BRAF cells comply with the association of mutation in the BRAF oncogene, with poorly differentiated carcinomas containing such structures and having poor patient survival (42-44). There are several types of KRAS mutations in CRCs associated with varying degrees of prognostic value (45), and only limited a number of studies have addressed the relationship of specific KRAS mutations with the histopathological structure of CRC. Interestingly, the KRAS codon 12 mutation used in the present study induced stronger anoikis resistance in fibroblastic NIH3T3 cells than codon 13 mutations (17). Along the lines of the role of anoikis resistance in metastasis, KRAS G12V mutations associated with poor prognosis unlike some other KRAS mutation types (45). Finally, KRAS G12V mutations were the second most common KRAS mutation type in serrated colorectal carcinoma (3), a carcinoma type characterized by MIPs. Our study, along with others, has shown an association of mutation in the KRAS oncogene with moderately/well-differentiated CRCs. This was visible in our 3D model with Caco2-KRAS cells where the cells either formed polarized cysts or partially filled structures, with some signs of apoptosis in cells within the luminal space $(46,47)$.

We were interested to review published data of colorectal cell lines with KRAS or BRAF mutations to see whether any association of 3D growth patterns and anoikis resistance could be detected (Table I). It is clearly evident (Table I) that both mutations associate with anoikis resistance and 3D growth as mainly solid clusters in both inherently mutated cell lines (HT-29 and SW-408) and in those transfected with mutations, as shown in the present study. Besides supporting the role of KRAS and BRAF mutations in the pathogenesis of anoikis resistance, these findings support the idea that anoikis resistance contributes to the formation of multicellular clusters with inner cells surviving without contact with the ECM. Finally, availability of cell lines harboring native KRAS G12V or BRAF V600E mutations would allow knock-out/knock in studies to confirm the importance of these mutations in anoikis resistance and the associated $3 \mathrm{D}$ growth characteristics.

The mechanisms behind the observed differences between BRAF and KRAS mutations in anoikis resistance and 3D growth patterns remain largely speculative at present. Both mutations present their effects on both 3D growth patterns (32) and anoikis resistance $(16,17,21)$ via activation of the RAS-RAF-ERK pathway. We observed evidence for upregulation of Bim protein in anoikis resistant cells induced by both mutations. Bim is mostly considered as a proapoptotic protein. Accordingly, in melanocytic cells BRAF mutation induces anoikis resistance by downregulating $\operatorname{Bim}(19,20)$, and in a colorectal carcinoma cell line with BRAF mutation (COLO205) repression of Bim inhibits apoptosis (48). However, in some cancer cells, Bim is overexpressed and has a pro-survival role. In this context, proapoptotic effect of Bim is blocked by formation of complexes with MCL-1 leaving pro-survival function active (49). MCL-1 has been recognized as an important factor in BRAF induced anoikis resistance in a CRC cell line (21), and such mechanism would be plausible in our transfected Caco- 2 cells. The differences in downstream signaling of mutated KRAS and BRAF molecules $(14,31,32)$ might further explain the observed differences in both anoikis resistance and 3D structure details, such as increased cell proliferation, altered apical-basal polarity establishment, disrupted integrin signaling, and disrupted intercellular contacts $(32,50)$.

Although monitoring of $3 \mathrm{D}$ structures by confocal imaging is efficient, it does not allow structural comparison with conventional formalin-fixed paraffin-embedded pathological specimens or the use of visible light immunohistochemical methods. This led us to use a simple yet robust and cost-efficient procedure for embedding 3D cell cultures in agar followed by fixing in formalin and embedding in paraffin blocks. This provided conventional tissue sections with well-preserved morphology. Accordingly, it was possible to compare well-preserved morphology in $3 \mathrm{D}$ cultures to that of human carcinoma samples and it is even possible to perform biomarker studies with immunohistochemical analyses.

Considering the benefits and importance of the current model, our 3D in vitro model is better than spheroid cultures in fluid (51), complemented with the presentation of maturation and layering of the cells on extracellular matrix as in tumor tissues. 3D culture allows live imaging, providing visualization of cell morphology and details during the formation of different structures with a well-maintained environment for the cells while imaging. Importantly, there have been no relevant models for studying anoikis resistance in CRC. However, relating with the lack of in vivo experiments in our study, more studies are clearly needed to gather further evidence whether formation of solid structures as seen in the present study is a manifestation of anoikis resistance. Furthermore, it would be essential to show in vivo importance of anoikis resistance induced by KRAS and BRAF mutations 
by xenotransplantation experiments. Such experiments might also provide information about occurrence of characteristic histopathological features of anoikis resistance in vivo.

We have shown that KRAS and BRAF mutations induce anoikis resistance in Caco-2 cells. In 3D cell culture, these mutations changed the growth of Caco- 2 cells drastically, from cyst formation to solid growth or focal intraluminal growth of cells. Both patterns represent morphology corresponding to the presentation of anoikis resistance in actual colorectal carcinomas, with inner cells surviving without contact with the extracellular matrix while outer cells survive based on their extracellular matrix contact. Accordingly, these mutant cell lines in 3D cultures serve, for example, in studies analyzing the potential need for specific therapeutic strategies against anoikis-resistant subpopulations of tumor cells. Besides anoikis resistance, KRAS mutation induced inversed polarity in Caco-2 cells, thus providing an in vitro model for this aberration, which is important in the dissemination of CRC (42).

\section{Acknowledgements}

The authors would like to thank Ms. Riitta Vuento (Department of Pathology, Oulu University Hospital, Oulu Finland, and Biobank Borealis, Oulu Univeristy Hospital and University of Oulu, Finland) for providing expertise in histopathological techniques and Dr. Virpi Glumoff (Faculty of Medicine, University of Oulu, Finland) for help in flow cytometric analyses.

\section{Funding}

The present study was funded by Finnish State Medical Research funding, allocated by the Oulu University Hospital, Finland.

\section{Availability of data and materials}

All data generated and analyzed during the present study are included in this published article.

\section{Authors' contributions}

TJK conceptualized and designed the study and drafted the manuscript. SE contributed to experimental design, analysis and revised the manuscript. MP designed the study, performed all the experiments, analyzed and drafted the manuscript. MJM and AT contributed to conception and design of the study and revised the manuscript. All authors reviewed and approved the final manuscript.

\section{Ethics approval and consent to participate}

Not applicable.

\section{Patient consent for publication}

Not applicable.

\section{Competing interests}

The authors declare that they have no competing interests.

\section{References}

1. Torre LA, Bray F, Siegel RL, Ferlay J, Lortet-Tieulent J and Jemal A: Global cancer statistics, 2012. CA Cancer J Clin 65: 87-108, 2015

2. Arrington AK, Heinrich EL, Lee W, Duldulao M, Patel S, Sanchez J, Garcia-Aguilar J and Kim J: Prognostic and predictive roles of KRAS mutation in colorectal cancer. Int J Mol Sci 13: 12153-12168, 2012.

3. Stefanius K, Ylitalo L, Tuomisto A, Kuivila R, Kantola T, Sirnio P, Karttunen TJ and Mäkinen MJ: Frequent mutations of KRAS in addition to BRAF in colorectal serrated adenocarcinoma. Histopathology 58: 679-692, 2011.

4. Neumann J,Zeindl-Eberhart E, Kirchner T and Jung A: Frequency and type of KRAS mutations in routine diagnostic analysis of metastatic colorectal cancer. Pathol Res Pract 205: 858-862, 2009.

5. Foltran L, De Maglio G, Pella N, Ermacora P, Aprile G, Masiero E, Giovannoni M, Iaiza E, Cardellino GG, Lutrino SE, et al: Prognostic role of KRAS, NRAS, BRAF and PIK3CA mutations in advanced colorectal cancer. Future Oncol 11: 629-640, 2015.

6. Guo F, Gong H, Zhao H, Chen J, Zhang Y, Zhang L, Shi X, Zhang A, Jin H, Zhang $\mathrm{J}$ and He Y: Mutation status and prognostic values of KRAS, NRAS, BRAF and PIK3CA in 353 chinese colorectal cancer patients. Sci Rep 8: 6076, 2018.

7. Matallanas D, Birtwistle M, Romano D, Zebisch A, Rauch J, von Kriegsheim A and Kolch W: Raf family kinases: Old dogs have learned new tricks. Genes Cancer 2: 232-260, 2011.

8. Yokota T, Ura T, Shibata N, Takahari D, Shitara K, Nomura M, Kondo C, Mizota A, Utsunomiya S, Muro K and Yatabe Y: BRAF mutation is a powerful prognostic factor in advanced and recurrent colorectal cancer. Br J Cancer 104: 856-862, 2011.

9. Lai E, Pretta A, Impera V, Mariani S, Giampieri R, Casula L, Pusceddu V, Coni P, Fanni D, Puzzoni M, et al: BRAF-mutant colorectal cancer, a different breed evolving. Expert Rev Mol Diagn 18: 499-512, 2018.

10. Gilmore AP: Anoikis. Cell Death Differ 12 (Suppl 2): S1473-S1477, 2005.

11. Guadamillas MC, Cerezo A and Del Pozo MA: Overcoming anoikis-pathways to anchorage-independent growth in cancer. J Cell Sci 124: 3189-3197, 2011.

12. Jinka R, Kapoor R, Sistla PG, Raj TA and Pande G: Alterations in cell-extracellular matrix interactions during progression of cancers. Int J Cell Biol 2012: 219196, 2012.

13. Frisch SM and Ruoslahti E: Integrins and anoikis. Curr Opin Cell Biol 9: 701-706, 1997.

14. Paoli P, Giannoni E and Chiarugi P: Anoikis molecular pathways and its role in cancer progression. Biochim Biophys Acta 1833: 3481-3498, 2013.

15. Demers MJ, Thibodeau S, Noël D, Fujita N, Tsuruo T, Gauthier R, Arguin $\mathrm{M}$ and Vachon PH: Intestinal epithelial cancer cell anoikis resistance: EGFR-mediated sustained activation of Src overrides Fak-dependent signaling to MEK/Erk and/or PI3-K/Akt-1. J Cell Biochem 107: 639-654, 2009.

16. Mason JA, Davison-Versagli CA, Leliaert AK, Pape DJ, McCallister C, Zuo J, Durbin SM, Buchheit CL, Zhang S and Schafer ZT: Oncogenic Ras differentially regulates metabolism and anoikis in extracellular matrix-detached cells. Cell Death Differ 23: 1271-1282, 2016.

17. Guerrero S, Casanova I, Farré L, Mazo A, Capellà G and Mangues R: K-ras codon 12 mutation induces higher level of resistance to apoptosis and predisposition to anchorage-independent growth than codon 13 mutation or proto-oncogene overexpression. Cancer Res 60: 6750-6756, 2000.

18. Jones RP, Sutton PA, Evans JP, Clifford R, McAvoy A, Lewis J, Rousseau A, Mountford R, McWhirter D and Malik HZ: Specific mutations in KRAS codon 12 are associated with worse overall survival in patients with advanced and recurrent colorectal cancer. Br J Cancer 116: 923-929, 2017.

19. Boisvert-Adamo K and Aplin AE: Mutant B-RAF mediates resistance to anoikis via bad and Bim. Oncogene 27: 3301-3312, 2008.

20. Goldstein NB, Johannes WU, Gadeliya AV, Green MR, Fujita M, Norris DA and Shellman YG: Active N-Ras and B-Raf inhibit anoikis by downregulating Bim expression in melanocytic cells. J Invest Dermatol 129: 432-437, 2009.

21. Kawakami H, Huang S, Pal K, Dutta SK, Mukhopadhyay D and Sinicrope FA: Mutant BRAF upregulates MCL-1 to confer apoptosis resistance that is reversed by MCL-1 antagonism and cobimetinib in colorectal cancer. Mol Cancer Ther 15: 3015-3027, 2016. 
22. Patankar M, Vayrynen S, Tuomisto A, Makinen M, Eskelinen S and Karttunen TJ: Micropapillary structures in colorectal cancer: An anoikis-resistant subpopulation. Anticancer Res 38: 2915-2921, 2018

23. Jia J, Zhang W, Liu JY, Chen G, Liu H, Zhong HY, Liu B, Cai Y, Zhang JL and Zhao YF: Epithelial mesenchymal transition is required for acquisition of anoikis resistance and metastatic potential in adenoid cystic carcinoma. PLoS One 7: e51549, 2012.

24. Fofaria NM and Srivastava SK: STAT3 induces anoikis resistance, promotes cell invasion and metastatic potential in pancreatic cancer cells. Carcinogenesis 36: 142-150, 2015.

25. Cai Q, Yan L and Xu Y: Anoikis resistance is a critical feature of highly aggressive ovarian cancer cells. Oncogene 34: 3315-3324, 2015.

26. Herrmann D, Conway JR, Vennin C, Magenau A, Hughes WE Morton JP and Timpson P: Three-dimensional cancer models mimic cell-matrix interactions in the tumour microenvironment. Carcinogenesis 35: 1671-1679, 2014.

27. Edmondson R, Broglie JJ, Adcock AF and Yang L: Three-dimensional cell culture systems and their applications in drug discovery and cell-based biosensors. Assay Drug Dev Technol 12: 207-218, 2014.

28. Weiswald LB, Bellet D and Dangles-Marie V: Spherical cancer models in tumor biology. Neoplasia 17: 1-15, 2015 .

29. Fang Y and Eglen RM: Three-dimensional cell cultures in drug discovery and development. SLAS Discov 22: 456-472, 2017.

30. Langhans SA: Three-dimensional in vitro cell culture models in drug discovery and drug repositioning. Front Pharmacol 9: 6, 2018.

31. Makrodouli E, Oikonomou E, Koc M, Andera L, Sasazuki T, Shirasawa $S$ and Pintzas A: BRAF and RAS oncogenes regulate rho GTPase pathways to mediate migration and invasion properties in human colon cancer cells: A comparative study. Mol Cancer 10: 118, 2011.

32. MagudiaK,Lahoz A and Hall A: K-Ras and B-Raf oncogenes inhibit colon epithelial polarity establishment through up-regulation of c-myc. J Cell Biol 198: 185-194, 2012.

33. Debnath J, Muthuswamy SK and Brugge JS: Morphogenesis and oncogenesis of MCF-10A mammary epithelial acini grown in three-dimensional basement membrane cultures. Methods 30 : 256-268, 2003

34. Camplejohn RS: Flow cytometric measurement of cell proliferation. Methods Mol Med 57: 133-143,2001.

35. Pinto MP, Jacobsen BM and Horwitz KB: An immunohistochemical method to study breast cancer cell subpopulations and their growth regulation by hormones in three-dimensional cultures. Front Endocrinol (Lausanne) 2: 15, 2011.

36. Kozlova NI, Morozevich GE, Chubukina AN and Berman AE: Integrin alphavbeta3 promotes anchorage-dependent apoptosis in human intestinal carcinoma cells. Oncogene 20: 4710-4717, 2001.

37. Cremolini C, Di Maio M, Petrelli F, Berenato R, Loupakis F and Pietrantonio F: BRAF-mutated metastatic colorectal cancer between past and future. Br J Cancer 113: 1634-1635, 2015

38. Orlandi A, Calegari A, Inno A, Berenato R, Caporale M, Niger M, Bossi I, Di Bartolomeo M, de Braud F and Pietrantonio F: BRAF in metastatic colorectal cancer: The future starts now. Pharmacogenomics 16: 2069-2081, 2015.

39. Pollan SG, Huang F, Sperger JM, Lang JM, Morrissey C, Cress AE, Chu CY, Bhowmick NA, You S, Freeman MR, et al: Regulation of inside-out $\beta 1$-integrin activation by CDCP1 Oncogene 37: 2817-2836, 2018.

40. Zhang X, Cromwell JW, Kunjummen BD, Yee D and Garcia-Aguilar J: The alpha2 and alpha3 integrins are required for morphologic differentiation of an intestinal epithelial cell line. Surgery 133: 429-437, 2003.

41. Zajac O, Raingeaud J, Libanje F, Lefebvre C, Sabino D, Martins I, Roy P, Benatar C, Canet-Jourdan C, Azorin P, et al: Tumour spheres with inverted polarity drive the formation of peritoneal metastases in patients with hypermethylated colorectal carcinomas. Nat Cell Biol 20: 296-306, 2018.
42. Clarke CN and Kopetz ES: BRAF mutant colorectal cancer as a distinct subset of colorectal cancer: Clinical characteristics, clinical behavior, and response to targeted therapies. J Gastrointest Oncol 6: 660-617, 2015

43. Jang MH, Kim S, Hwang DY, Kim WY, Lim SD, Kim WS, Hwang TS and Han HS: BRAF-mutated colorectal cancer exhibits distinct clinicopathological features from wild-type BRAF-expressing cancer independent of the microsatellite instability status. J Korean Med Sci 32: 38-46, 2017.

44. $\mathrm{Li} \mathrm{Y}$ and $\mathrm{Li} \mathrm{W}$ : BRAF mutation is associated with poor clinicopathological outcomes in colorectal cancer: A meta-analysis. Saudi J Gastroenterol 23: 144-149, 2017.

45. Modest DP, Ricard I, Heinemann V, Hegewisch-Becker S, Schmiegel W, Porschen R, Stintzing S, Graeven U, Arnold D, von Weikersthal LF, et al: Outcome according to KRASNRAS- and BRAF-mutation as well as KRAS mutation variants: Pooled analysis of five randomized trials in metastatic colorectal cancer by the AIO colorectal cancer study group. Ann Oncol 27: 1746-1753, 2016

46. Fu T, Guzzetta AA, Jeschke J, Vatapalli R, Dave P, Hooker CM, Morgan R, Iacobuzio-Donahue CA, Liu B and Ahuja N: KRAS $\mathrm{G}>\mathrm{A}$ mutation favors poor tumor differentiation but may not be associated with prognosis in patients with curatively resected duodenal adenocarcinoma. Int J Cancer 132: 2502-2509, 2013.

47. Margonis GA, Kim Y, Spolverato G, Ejaz A, Gupta R, Cosgrove D, Anders R, Karagkounis G, Choti MA and Pawlik TM: Association between specific mutations in KRAS codon 12 and colorectal liver metastasis. JAMA Surg 150: 722-179, 2015.

48. Wickenden JA, Jin H, Johnson M, Gillings AS, Newson C, Austin M, Chell SD, Balmanno K, Pritchard CA and Cook SJ: Colorectal cancer cells with the BRAF(V600E) mutation are addicted to the ERK1/2 pathway for growth factor-independent survival and repression of BIM. Oncogene 27: 7150-7161, 2008.

49. Gogada R, Yadav N, Liu J, Tang S, Zhang D, Schneider A, Seshadri A, Sun L, Aldaz CM, Tang DG and Chandra D: Bim, a proapoptotic protein, up-regulated via transcription factor E2F1-dependent mechanism, functions as a prosurvival molecule in cancer. J Biol Chem 288: 368-381, 2013.

50. Teräväinen TP, Myllymäki SM, Friedrichs J, Strohmeyer N, Moyano JV, Wu C, Matlin KS, Muller DJ and Manninen A: $\alpha \mathrm{V}$-integrins are required for mechanotransduction in MDCK epithelial cells. PLoS One 8: e71485, 2013.

51. Ferreira LP, Gaspar VM and Mano JF: Design of spherically structured 3D in vitro tumor models-advances and prospects. Acta Biomater 75: 11-34, 2018.

52. Stevens PD, Wen YA, Xiong X, Zaytseva YY, Li AT, Wang C, Stevens AT, Farmer TN, Gan T, Weiss HL, et al: Erbin suppresses KSR1-mediated RAS/RAF signaling and tumorigenesis in colorectal cancer. Cancer Res 78: 4839-4852, 2018.

53. Riedl A, Schlederer M, Pudelko K, Stadler M, Walter S, Unterleuthner D, Unger C, Kramer N, Hengstschlager M, Kenner L, et al: Comparison of cancer cells in 2D vs 3D culture reveals differences in AKT-mTOR-S6K signaling and drug responses. J Cell Sci 130: 203-218, 2017.

54. Luca AC, Mersch S, Deenen R, Schmidt S, Messner I, Schäfer KL, Baldus SE, Huckenbeck W, Piekorz RP, Knoefel WT, et al: Impact of the 3D microenvironment on phenotype, gene expression, and EGFR inhibition of colorectal cancer cell lines. PLoS One 8: e59689, 2013.

55. Derouet M, Wu X, May L, Hoon Yoo B, Sasazuki T, Shirasawa S, Rak J and Rosen KV: Acquisition of anoikis resistance promotes the emergence of oncogenic K-ras mutations in colorectal cancer cells and stimulates their tumorigenicity in vivo. Neoplasia 9: 536-545, 2007

his work is licensed under a Creative Commons Attribution 4.0 International (CC BY 4.0) License. 